\title{
Evolving P450pyr Monooxygenase for Regio- and Stereoselective Hydroxylations
}

\author{
Yi Yang and Zhi Li*
}

\begin{abstract}
P450pyr monooxygenase from Sphingomonas sp. HXN-200 catalysed the regio- and stereoselective hydroxylation at a non-activated carbon atom, a useful but challenging reaction in classic chemistry, with unique substrate specificity for a number of alicyclic compounds. New P450pyr mutants were developed by directed evolution with improved catalytic performance, thus significantly extending the application of the P450pyr monooxygenase family in biohydroxylation to prepare useful and valuable chiral alcohols. Directed evolution of P450pyr created new enzymes with improved $S$-enantioselectivity or $R$-enantioselectivity for the hydroxylation of $N$-benzyl pyrrolidine, enhanced regioselectivity for the hydroxylation of $N$-benzyl pyrrolidinone, and increased enantioselectivity for the hydroxylation of $N$-benzyl piperidinone, respectively. Directed evolution of P450pyr generated also mutants with fully altered regioselectivity (from terminal to subterminal) and newly created excellent $S$-enantioselectivity for the biohydroxylation of $n$-octane and propylbenzene, respectively, providing new opportunities for the regio- and enantioselective alkane functionalization. New P450pyr mutants were engineered as the first catalyst for highly selective terminal hydroxylation of $n$-butanol to 1,4 -butanediol. Several novel, accurate, sensitive, simple, and HTS assays based on colorimetric or MS detection for measuring the enantio- and/or regioselectivity of hydroxylation were developed and proven to be practical in directed evolution. The P450pyr X-ray structure was obtained and used to guide the evolution. In silico modelling and substrate docking provided some insight into the influence of several important amino acid mutations of the engineered P450pyr mutants on the altered or enhanced regio- and enantioselectivity as well as new substrate acceptance. The obtained information and knowledge is useful for further engineering of P450pyr for other hydroxylations and oxidations.
\end{abstract}

Keywords: Biocatalysis · C-H activation · Directed evolution · Hydroxylation · P450 monooxygenase

\section{Introduction}

Regio- and stereoselective hydroxylations are very important in green chemistry as well as in chemical and pharmaceutical synthesis. ${ }^{[1]}$ Despite of some progress, this type of reaction has been remained a challenge for classic chemistry. ${ }^{[2]}$ Alternatively, nature provides a useful solution for the hydroxylations by using a monooxygenase as catalyst and molecular oxygen as the cheap and green oxidant. ${ }^{[3]}$ Many monooxygenases are reported for hydroxylations, ${ }^{[4]}$ and cytochrome P450 monooxygenases form the largest subfamily. Some P450s are known to catalyse the hydroxylations of special types of substrates, with high chemical-, regio- and/or stereoselectivity. ${ }^{[5]}$ For example, P450cam from Pseudomonas putida, in the presence of ferredoxin ( $\mathrm{Fdx})$ and ferredoxin reductase (FdR), catalysed the highly selective oxidation of camphor to give 5-exo-hydroxy-camphor. ${ }^{[6]}$

${ }^{*}$ Correspondence: Prof. Z. Li

National University of Singapore

Department of Chemical \& Biomolecular Engineering 4 Engineering Drive 4, Singapore 117585, Singapore

Tel: +6565168416

E-mail: chelz@nus.edu.sg
P450BM3 from Bacillus megaterium, a self-sufficient P450, catalysed the hydroxylation of several long-chain fatty acids, amides, and alcohols with high activity at $\omega-1, \omega-2$, and $\omega-3$ positions. ${ }^{[7]}$ So far, over 7,000 P450s have been identified and sequenced, many soluble bacterial P450s have been overexpressed in heterologous hosts for synthesis, and some of them are characterized with 3D structures. ${ }^{[8]}$

There are still problems that limit the wide application of P450 monooxygenases for regio- and stereoselective hydroxylations, such as the narrow substrate scope and unsatisfactory activity and selectivity towards a given non-natural substrate. Since the 1990s, directed evolution has become a powerful tool to create new enzymes with better catalytic performance. ${ }^{[9]}$ By using this method, several P450 mutant monooxygenases were obtained with new substrate acceptance, ${ }^{[10]}$ improved activity, ${ }^{[11]}$ and increased regio- and/or stereo-selectivity ${ }^{[12]}$ for certain targeted hydroxylations. For instance, new P450cam and P450BM3 mutants were generated for the hydroxylation of polycyclic aromatic hydrocarbons with enhanced activity ${ }^{[1]}$ and the hydroxylation of testosterone ${ }^{[13]}$ and artemisinin ${ }^{[14]}$ with enhanced regioand diastereoselectivity. Nevertheless, new P450 monooxygenases with unique substrate specificity and high regio- and stereoselectivity are highly desirable for hydroxylations in practical synthesis.

We previously identified a novel P450pyr monooxygenase from Sphingomonas sp. HXN-200 as a class I P450 system consisting of P450pyr, Fdx, and FdR for the biohydroxylation of several alicyclic compounds. ${ }^{[15]}$ It was the best known catalyst for the hydroxylation of $N$-substituted pyrrolidines, azetidines, piperidines, 2-piperidinones, and 2-pyrrolidinones, with good activity, high chemoand regioselectivity, and good to excellent enantioselectivity. ${ }^{[15]}$ We recently further engineered P450pyr by directed evolution to develop new catalysts for hydroxylations (Scheme 1) with enhanced or altered regio- and stereoselectivity as well as new substrate specificity, providing more prac-

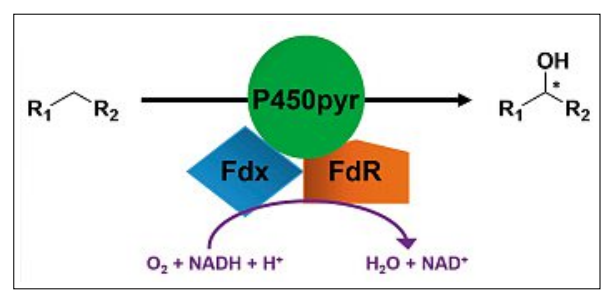

Scheme 1. Regio- and stereoselective hydroxylations with P450pyr monooxygenase. stereoselectivity, and substrate acceptance. 
tical or new synthetic applications of biohydroxylations.

\section{Engineering of P450pyr Monooxygenase for R-Enantioselective Hydroxylation of $\mathbf{N}$-Benzyl-pyrrolidine}

The biohydroxylation of $N$-benzylpyrrolidine 1 to give either $(R)$ - or $(S)$ $N$-benzyl-3-hydroxypyrrolidines 2 (Table $1)$ is very interesting, since both $(R)-2$ and $(S)-\mathbf{2}$ are useful intermediates for the preparation of carbapenem antibiotics, a $k$-receptor agonist, a 5-HT $\mathrm{HT}_{1 \mathrm{a}}$ receptor agonist, and an antibacterial agent. ${ }^{[16]} \mathrm{P} 450 \mathrm{pyr}$ monooxygenase was the only known enzyme for this hydroxylation, but gave the product in only $53 \%$ ee $(S)$. Thus, directed evolution of P450pyr was performed to create more enantioselective enzymes for the hydroxylation. ${ }^{[17]}$

A P450pyr homology model was first built based on the crystal structures of CYP119 and P450st. ${ }^{[18]}$ Seventeen amino acid residues, which are located within $5 \AA$ of the heme-substrate in the catalytic pocket, were identified for iterative saturation mutagenesis (ISM). ${ }^{[19]}$ An E. coli BL21(DE3) strain was constructed to contain dual plasmids (pRSFDuet P450pyr and pETDuet Fdx FdR) for co-expressing P450pyr, Fdx, and FdR, giving high activity for the target hydroxylation. This system was used to generate whole-cell catalysts expressing P450pyr mutants for the screening of enantioselective biohydroxylation.

The development of an applicable high-throughput screening (HTS) assay for measuring enantioselectivity is the key challenge in evolving enantioselective enzymes. ${ }^{[20]}$ Most of the reported directed evolution of enantioselective enzymes focused on kinetic resolution, and very few involved asymmetric reactions of a prochiral substrate due to lack of an appropriate $e e$-assay. We previously developed a novel concept of utilising two complementary alcohol dehydrogenases to determine the $e e$ value of a chiral alcohol.[21] NBT-PMS colorimetric assay was then introduced to make this concept more easily applicable in directed evolution (Scheme 2A). Two alcohol dehydrogenases BRD ${ }^{[22]}$ and $\mathrm{RDR}^{[23]}$ (from Micrococcus luteus and Devosia riboflavina, respectively) were identified for the specific oxidation of $(S)$-2 and $(R)-2$, respectively, and they were used in the colorimetric HTS assay. The ee of the hydroxylation product 2 was estimated by comparison of the production of formazan (colorimetric or $\mathrm{UV}_{580}$ ) of the two parallel oxidations of the product 2 with BRD and RDR, respectively. ${ }^{[24]}$ The assay was first validated with mixtures containing $(S)-\mathbf{2}$ and $(R)-\mathbf{2}$ at different ratios and then applied in the real evolution to screen P450pyr mutants.

Table 1. Regio- and stereoselective hydroxylations with E. coli cells co-expressing P450pyr or its mutants with Fdx and FdR

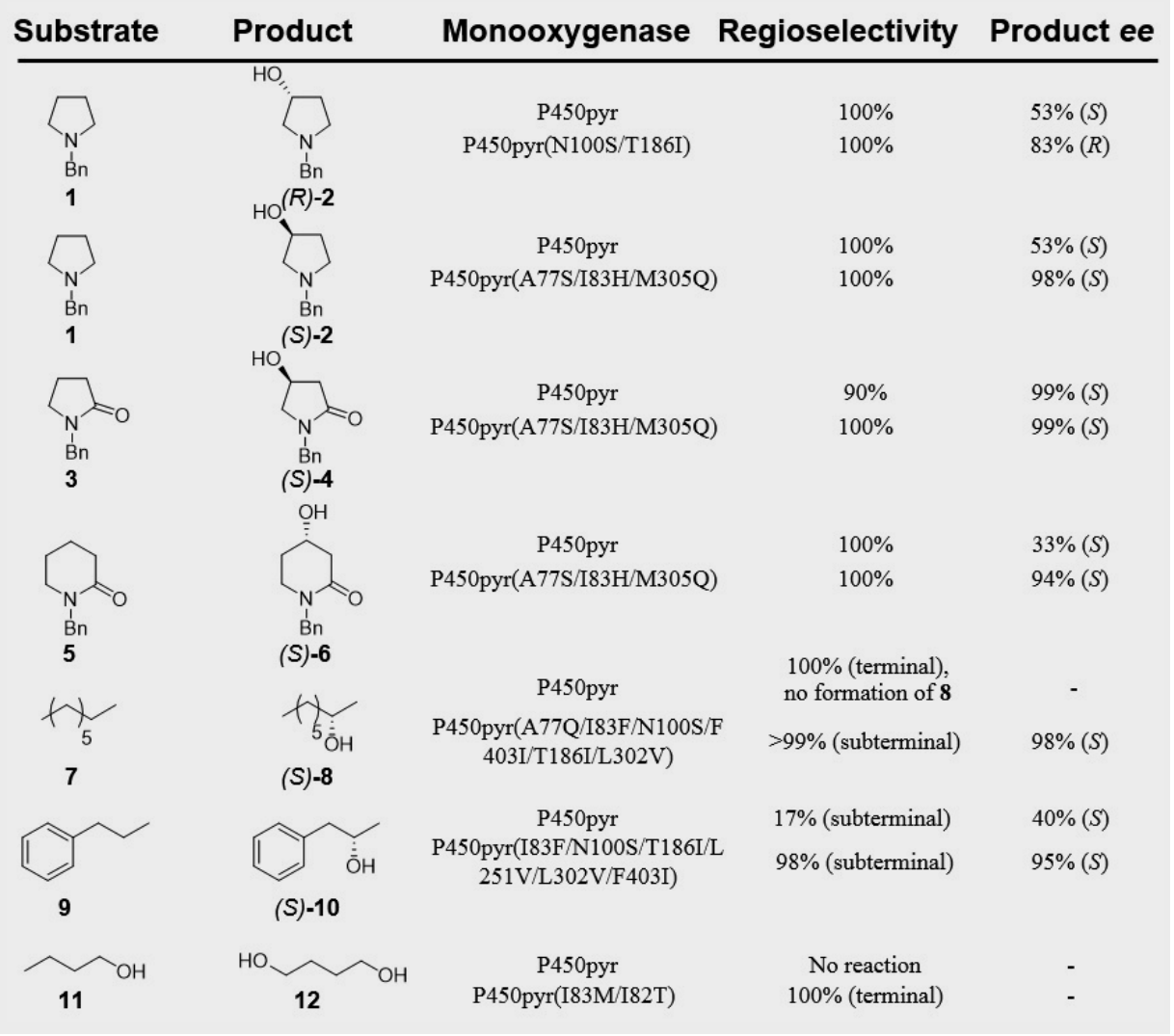

After screening 3060 clones in the $1^{\text {st }}$ round of evolution, mutant 1AF4 (N100S) was found to show the inverted enantioselectivity to give $(R)-2$ in $42 \%$ ee . P450pyrN100S was used as a template in the $2^{\text {nd }}$ round of evolution. Four mutants were identified with improved $(R)$ enantioselectivity, and the best mutant 1AF4A (N100S, T186I) produced $(R)-2$ in $83 \%$ ee (Table 1). This is an interesting example of inverting hydroxylation enantioselectivity of a P450 monooxygenase by directed evolution. This is also the first example of successfully evolving a class I P450 monooxygenase with enhanced enantioselectivity in asymmetric hydroxylation.

\section{Engineering P450pyr Monooxygenase for Highly S-Enantioselective Hydroxylation of $N$-Benzyl-pyrrolidine}

Directed evolution of P450pyr was also performed to obtain enzyme for the highly $S$-enantioselective biohydroxylation of $\mathbf{1}$ to product $(S)-2$ in $\geq 98 \%$ ee. It was very challenging to engineer an enantioselective monooxygenase for biohydroxylation to achieve product $e e$ of $\geq 98 \%$, a necessary $e e$ value of a chiral intermediate in pharmaceutical manufacturing. To achieve this, the 3D structure (PBD ID 3RWL) of histagged P450pyr was first obtained by X-ray diffraction. ${ }^{[25]}$ Based on the structure, 20 residues that are located either in the active site vicinity or in the gorge accessing the heme were chosen for ISM. A novel, sensitive, accurate, and high-throughput screening assay for determining the enantioselectivity of biohydroxylation was developed for the evolution by using two enantiomers of isotopically labelled substrate for parallel enzymatic hydroxylations, coupled with MS detection.

The principle is shown in Scheme 2B. $(S)$-D-1 and ( $R$-D-1 are prepared and used as substrates for the hydroxylation. The $\mathrm{O}-\mathrm{D}$ bond in the hydroxylation product changes quickly to $\mathrm{O}-\mathrm{H}$ in the aqueous medium to give a mass of $\mathrm{M}$ for $(S)-2$ and $(R)-2$ while other hydroxylation products still have a mass of $M+1$. The peak ratio of $\mathrm{M}$ and $\mathrm{M}+1$ in $\mathrm{MS}$ can be easily determined and then used to calculate the product $e e$ of the hydroxylation of $\mathbf{1}$ based on Eqn. (1) and (2) (Scheme 2B). ${ }^{26]}$ The assay was validated for Sphingomonas sp. HXN-200-catalysed hydroxylation of 1 by using LC-MS analysis and compared with chiral HPLC analysis. The MS-assay gives high accuracy for the determination of the product $e e$, allows for direct assaying the aqueous sample of the biotransformation mixtures, does not require separation in LC-MS analysis, shows high sensitivi- 


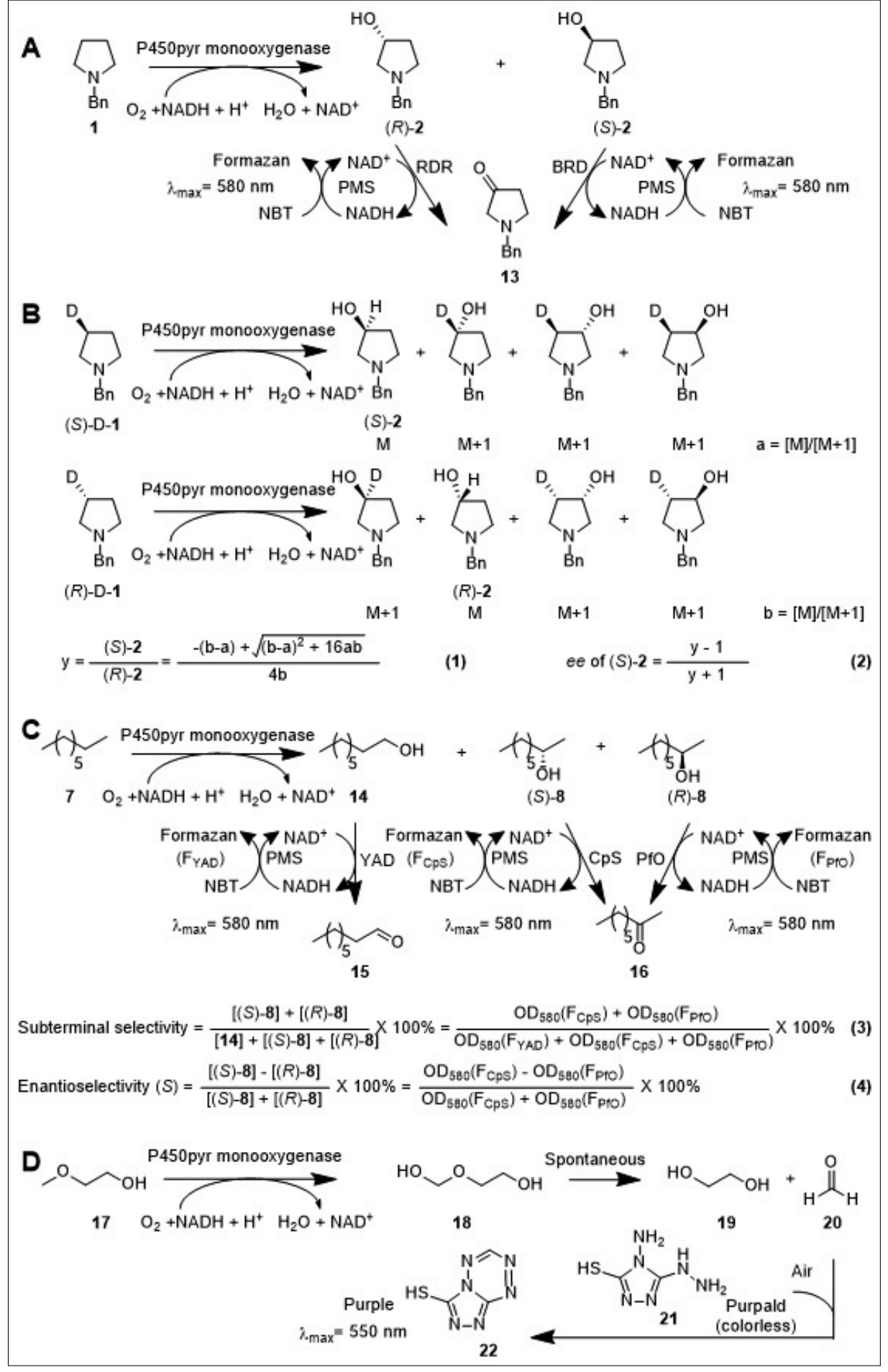

Scheme 2. Principles of the HTS assays used for directed evolution of P450pyr. A) Two-enzyme based colorimetric ee assay for asymmetric biohydroxylation of $N$-benzyl-pyrrolidine 1 to $(R)$ and (S)-2; B) Mass spectrometry based ee assay for asymmetric biohydroxylation of $N$-benzylpyrrolidine 1 to $(R)$ - and (S)-2; C) Three-enzyme based colorimetric assay to determine subterminal selectivity and enantioselectivity for the hydroxylation of $n$-octane 7 to $(R)$ - and (S)-8. D) Surrogate substrate $\mathbf{1 7}$ based colorimetric HTS assay to determine the terminal hydroxylation activity for the hydroxylation of $n$-butanol $\mathbf{1 1}$

ty (detectable for $0.005 \mathrm{mM}$ of product), takes $1.0 \mathrm{~min}$ for analysis, and provides a theoretical throughput analysis of 1440 samples per day.

By using the MS-based HTS ee assay, several more enantioselective mutants were identified from the evolution. The ee values of the hydroxylation product $\mathbf{2}$ with these mutants were further determined by chiral HPLC analysis, being nearly the same as the values measured from the MSbased assay.

After screening only 5264 colonies in two rounds of evolution, mutant $\mathrm{I} 83 \mathrm{H} /$ M305Q was identified to give product $e e$ of $94 \%(S)$. In the $3^{\text {rd }}$ round of evolution, a triple mutant P450pyrTM (I83H/M305Q/ A77S) was identified to produce $(S)-\mathbf{2}$ in
98\% ee. P450pyrTM showed a specific activity of $5.2 \mathrm{U} / \mathrm{g} \mathrm{cdw}$, being $81 \%$ of the activity of P450pyr. It also gave a clean hydroxylation with no other byproducts. Compared with P450pyr, P450pyrTM demonstrated slightly higher values of apparent $k_{\text {cat }}$ and $K_{\mathrm{m}}$ (26.9 vs $21.9 \mathrm{~min}^{-1}$ and 15.6 vs $13.6 \mathrm{mM}$, respectively) and similar catalytic efficiency $\left(k_{\text {cat }} / K_{\mathrm{m}}\right)$. This is an unique example of engineering a highly enantioselective monooxygenase via directed evolution for asymmetric biohydroxylation while maintaining high catalytic activity.

Mutant P450pyrTM was examined for the hydroxylation of other substrates. In comparison with wild-type P450pyr, P450pyrTM improved the regioselectivity from $90 \%$ to $100 \%$ for the hydroxylation of $N$-benzyl-pyrrolidinone 3 to $(S)$ 4-hydroxy- $N$-benzyl-pyrrolidinone $\mathbf{4}$ and enhanced the enantioselectivity for the hydroxylation of $N$-benzylpiperidinone $\mathbf{5}$ to $(S)$-4-hydroxy- $N$ - benzylpiperidinone $\mathbf{6}$ in 94\% ee from 33\% ee (Table 1).[27]

Interestingly, P450pyrTM also catalysed the asymmetric epoxidation of some para-substituted styrenes, ${ }^{[28]}$ being the first enzyme to give high $R$-enantioselectivity as well as high activity and conversion. P450pyrTM showed broad substrate range for asymmetric epoxidation and showed also high enantioselectivity for the epoxidations of 2-methyl-3-phenyl-1-propene (an unconjugated 1,1-disubstituted alkene) and $N$-phenoxycarbonyl-1,2,5,6tetrahydropyridine (a cyclic alkene), respectively. [28]

The single mutant $\mathrm{P} 450$ pyrI83H in an aqueous/ionic liquid (IL) biphasic system showed the best $R$-enantioselectivity and activity known thus far for the sulfoxidation of thioanisole, 4-fluoro-thioanisole, ethyl phenyl sulfide, to give $(R)$-phenyl methyl sulfoxide in $99 \%$ ee, $(R)-4-$ fluorophenyl methyl sulfoxide in $98 \% e e$, and $(R)$-ethyl phenyl sulfoxide in $96 \% e e$, respectively. [29]

\section{Engineering of P450pyr Monooxygenase for Highly Regio- and Enantioselective Subterminal Hydroxylation of $n$-Octane.}

Regio- and stereoselective biohydroxylation at a non-activated $\mathrm{C}$-atom is very useful for the selective alkane functionalization, ${ }^{[30]}$ an easily available and abundant feedstock. Despite some progress on the discovery and engineering of enzymes for biohydroxylation, development of an enzyme for non-terminal alkane hydroxylation with very high regio- and enantioselectivity is still problematic: many known monooxygenases such as alkB, sMMO, and P450pyr demonstrated terminal selec- 
tivity; [31] P450BM-3 and P450 CYP102A3 gave a mixed $\omega-1, \omega-2$, and $\omega-3$ selectivity; ${ }^{[32]}$ several mutants of sMMO and alkB were engineered to show moderate subterminal selectivity; ${ }^{[33]}$ a P450BM-3 mutant was reported to catalyse the subterminal hydroxylation of $n$-octane 7 with $89 \%$ regioselectivity and $65 \%$ enantioselectivity; ${ }^{[34]}$ and $\mathrm{P} 450 \mathrm{cam}$ mutants were generated for subterminal hydroxylation of $n$-butane ${ }^{[35]}$ but with no report of product $e e$ values.

We succeeded in engineering terminal selective P450pyr monooxygenase for subterminal hydroxylation of $n$-octane 7 with high regio- and enantioselectivity to produce $(S)$-2-octanol 8, a useful intermediate for preparing high-performance liquid crystals ${ }^{[36]}$ and lipoprotein-associated phospholipase $\mathrm{A}_{2}\left(\mathrm{Lp}-\mathrm{PLA}_{2}\right.$ ) inhibitor for heart disease treatment. ${ }^{[37]}$ Based on the $n$-octane-P450pyr docking model, 22 amino acids residues within $6 \AA$ of $n$-octane, in the accessing channel, and in the 'big loop' were chosen for ISM. The screening of positive mutants was carried out with a novel colorimetric HTS assay to detect both regioselectivity and enantioselectivity for the hydroxylation of $n$-octane 7 at the subterminal position.

As illustrated in Scheme 2C, three alcohol dehydrogenases $\mathrm{CpSADH}$, PfODH, and YAD (from Candida parapsilosis, ${ }^{[38]}$ Pichia finlandica, ${ }^{[39]}$ and Saccharomyces cerevisiae, ${ }^{40]}$ respectively), which are highly specific for the oxidation of $(S)$ $\mathbf{8 ,}(R)-\mathbf{8}$, and 14, respectively, are used separately in three parallel experiments containing NBT, NAD ${ }^{+}$, and PMS, to catalyse the oxidation of the product mixtures from biohydroxylation of $n$-octane 7 . The concentrations of $(S)-\mathbf{8},(R)-\mathbf{8}$, and $\mathbf{1 4}$ are determined by analysing the formation of formazan with UV at $580 \mathrm{~nm}$. The subterminal selectivity and enantioselectivity of the biohydroxylation can be then calculated using Eqn. (3) and (4) (Scheme 2C), respectively. This HTS ee assay was validated by using mixtures of $(S)-\mathbf{8},(R)-\mathbf{8}$, and 14 at different ratio with high accuracy and sensitivity, independent of the total alcohol concentration.

By six rounds of evolution using the HTS assay, a sextuple mutant P450pyrSM1 (A77Q/I83F/N100S/F403I/T186I/L302V) was created for the subterminal hydroxylation of $n$-octane 7 to give $>99 \%$ subterminal selectivity, $98 \%(S)$-enantioselectivity, and $90 \%$ of the activity of P450pyr-catalysed terminal hydroxylation. With a $K$ of 2.187 $\mathrm{mM}$ and a $k_{\text {cat }}$ of $5.9 \mathrm{~min}^{-1}, \mathrm{P} 450 \mathrm{pyrSM} 1$ showed nearly the same catalytic efficiency $\left(k_{c a t} / K_{m}\right)$ for the subterminal hydroxylation as that of P450pyr for the terminal hydroxylation. P450pyrSM1 is the first catalyst for this type of highly subterminal selective and enantioselective hydroxylation of alkane. This is also the first example of fully altering enzyme regioselectivity and gaining high enantioselectivity at the same time for biohydroxylation by directed evolution.

The positive mutants created in the $6^{\text {th }}$ round of evolution were further screened for the subterminal hydroxylation of propylbenzene 9 to produce $(S)$-1-phenyl-2propanol 10, which is a pharmaceutical intermediate for preparing Amphetamine, ${ }^{[41]}$ (R)-Selegiline, ${ }^{[42]}$ and Cathepsin $\mathrm{K}$ inhibitors. ${ }^{[43]} \mathrm{A}$ sextuple mutant P450pyrSM2 (I83F/N 100S/T 186I/L 251 V/L 302V/ F403I) was found to catalyse the highly subterminal selective and $S$-enantioselective hydroxylation of 9 to give $(S)$-1-phenyl-2propanol 10 in $95 \%$ ee. No other enzymes were reported for this hydroxylation with high regio- and enantioselectivity. ${ }^{[34,44]}$

\section{Engineering of P450pyr Monooxygenase for Highly Regioselective Terminal Hydroxylation of $\boldsymbol{n}$-Butanol}

P450pyr showed excellent terminal hydroxylation toward hydrophobic substrates such as alkanes, but no activity towards hydrophilic molecules such as alcohols. Thus, P450pyr was engineered for the terminal hydroxylation of $n$-butanol 11 to produce 1,4-butanediol 12, a useful chemical in polymer synthesis and chemical production. ${ }^{[45]}$ This could provide a bio-based synthesis of 1,4-butanediol 12 from $n$-butanol 11 that is obtained by fermentation. No chemical- or biocatalyst has been reported so far for this hydroxylation.

A colorimetric HTS assay was developed for the evolution by using 2-methoxyethanol 17, which is structurally similar to $n$-butanol 11, as a surrogate substrate. As shown in Scheme 2D, hydroxylation of 2-methoxyethanol $\mathbf{1 7}$ at the terminal position gives the instable diol $\mathbf{1 8}$ which decomposes to ethylene glycol 19 and formaldehyde 20. The concentration of formaldehyde $\mathbf{2 0}$ in the reaction mixture can be determined by adding purpald 21 to produce a purple compound $\mathbf{2 2}$ and measuring the UV absorption at $550 \mathrm{~nm} .{ }^{[46]}$ The assay was proven sensitive and high-throughput, and it was used for the evolution.

Based on the 3D structure of P450pyr hydroxylase, 22 amino acid residues were chosen for ISM. The positive mutants selected by using the surrogate substrate-based HTS assay were further examined for biohydroxylation of $n$-butanol 11 . In the two rounds of evolution, P450pyr single mutant I83M and P450pyr double mutant I83M/I82T were found to show excellent terminal regioselectivity for the hydroxylation of $n$-butanol 11, with relatively higher activity and no byproduct formation. This gives an unique example of engineering a hydroxylase to accept a hydrophilic substrate from the original preference of a hydrophobic substrate by directed evolution. ${ }^{[47]}$ Biohydroxylations of $n$-butanol $\mathbf{1 1}$ and surrogate substrate 17 with these mutants, respectively, gave similar product concentration, which further confirmed the applicability of the surrogate substrate-based colorimetric HTS assay for this evolution.

The engineered P450pyr mutants are not active enough for practical hydroxylation. Nevertheless, it offers a solid basis for further development of more active enzymes for this hydroxylation.

\section{Molecular Basis for Improved Catalytic Performance of Engineered P450pyr Mutants}

In silico modelling and substrate docking has given some insight into the molecular basis of the observed change of regioselectivity and/or enantioselectivity as well as new substrate acceptance of the engineered P450pyr mutants. The key amino acid residues where the mutations lead to the improved catalytic performance are shown in the active site of the X-ray structure of P450pyr in Fig. 1.

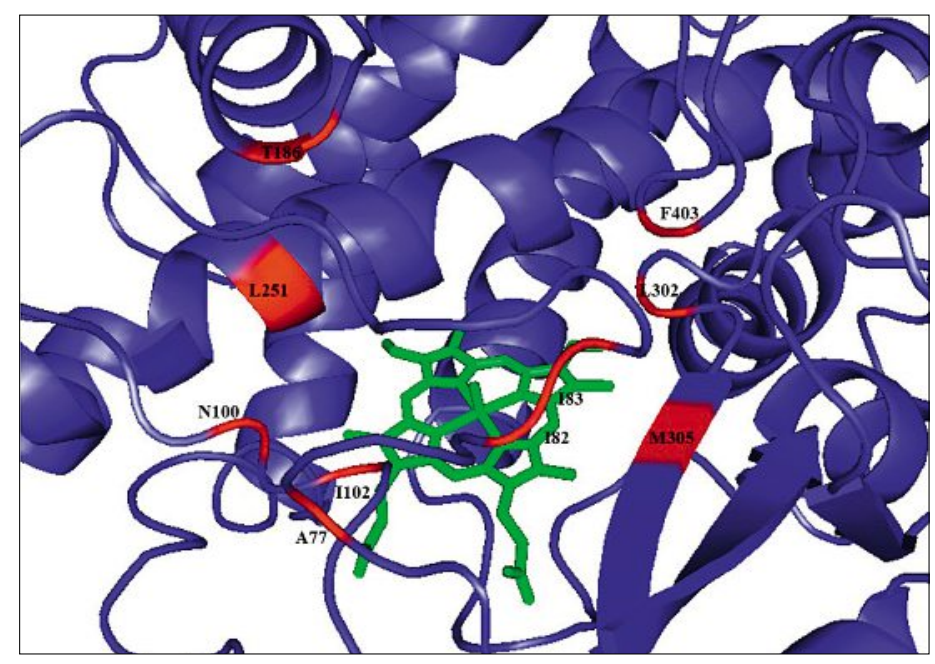

Fig. 1. The structure of P450pyr active site. Green: heme; red: key amino acid residues responsible for the improved catalytic performance through mutations at these positions 
For the hydroxylation of $N$-benzyl pyrrolidine 1 with P450pyr, the benzene ring of the substrate could be stabilized by three hydrophobic amino acids residues I82, I102, and L251, and its pyrrolidine ring is weakly interacted with L302. In the triple mutant P450pyrTM (A77S/I183H/ M305Q), the mutations of A77S and $\mathrm{I} 83 \mathrm{H}$ result in the formation of hydrogen bonds and move the big loop closer to the heme. ${ }^{[25]}$ The M305Q mutation also moves amino acid L302 closer to the heme. In P450pyrTM, the benzene ring of substrate $\mathbf{1}$ is located within a hydrophobic cleft above the heme and the pyrrolidine ring is restrained in the vicinity of the heme, thus creating the enhanced preference for $S$-enantioselective hydroxylation. ${ }^{25]}$

For P450pyr-catalysed hydroxylation of $n$-octane 7 , the substrate-enzyme binding pocket is very compact, and the hydrophobic substrate $n$-octane 7 has to take a vertical accessing pose to the heme, leading to terminal hydroxylation. For P450pyrSM1catalysed hydroxylation of $n$-octane 7, the binding pocket is reshaped. F403I and L302V mutations disrupt the hydrophobic cluster and extend the substrate-enzyme binding pocket. The substrate $n$-octane 7 adopts a horizontal posture over the heme to give a clear preference for $S$-selective subterminal hydroxylation. ${ }^{[44]}$

With $n$-butanol 11 in P450pyr, the substrate hydrophobic end is located between two hydrophobic amino acids residues I102 and L302, and the substrate hydroxyl group is oriented towards the hydrophilic amino acid residue $\mathrm{T} 259$ and close to heme-O. This posture is not suitable for any hydroxylation reaction. For P450pyr mutant I83M/I82T, I83M mutation provides with a longer amino acid residue $M$ and results in the decrease of the distance between I102 and L302. I82T mutation introduces a hydrophilic amino acid residue $\mathrm{T}$ and destroys the hydrophobic interaction between I82 and I102. Thus, $n$-butanol 11 takes a binding pose with the hydroxyl group away from heme-O and the hydrophobic end close to heme-O, thus enabling terminal hydroxylation. ${ }^{[47]}$

\section{Conclusion}

P450pyr monooxygenase is a useful enzyme with unique substrate specificity for regio- and stereoselective biohydroxylation at non-activated $\mathrm{C}$-atom, a challenging reaction in classic chemistry. By directed evolution, some P450pyr mutants were successfully created as much better enzymes for several selected target hydroxylations, with altered regioselectivity, enhanced $S$ - or $R$-enantioselectivity, or new substrate acceptance.

A P450pyr double mutant N100S/
T86I was created with inverted enantioselectivity for the hydroxylation of $N$-benzyl pyrrolidine 1 to produce $(R)-3$ hydroxypyrrolidine $\mathbf{2}$ in $83 \%$ ee.

A P450pyr triple mutant P450pyrTM (I83H/M305Q/A77S) was also developed for the highly enantioselective hydroxylation of $N$-benzyl pyrrolidine 1 to produce (S)-3-hydroxypyrrolidine $\mathbf{2}$ in $98 \%$ ee. P450pyrTM catalysed the hydroxylation of $N$-benzyl-pyrrolidinone 3 to give $(S)-4-$ hydroxy- $N$-benzyl-pyrrolidinone 4 in $99 \%$ ee with improved regioselectivity of $100 \%$. Moreover, P450pyrTM showed enhanced enantioselectivity for the hydroxylation of $\mathrm{N}$-benzylpiperidinone $\mathbf{5}$ to produce $(S)$-4-hydroxy- $N$-benzylpiperidinone $\mathbf{6}$ in 94\% ee.

Evolution of the terminal selective P450pyr afforded a P450pyr sextuple mutant P450pyrSM1 (A77Q/I83F/N100S/ F403I/T186I/L302V) for the subterminal hydroxylation of $n$-octane 7 with excellent regio- and enantioselectivity to produce $(S)$-2-octanol 8 in $98 \%$ ee. Another $\mathrm{P} 450$ pyr sextuple mutant P450pyrSM2 (I83F/N100S/T 186I/L251 V/L302V/ F403I) was also engineered to catalyse the subterminal hydroxylation of propylbenzene 9 to give $(S)$-1-phenyl-2-propanol 10 in $95 \% e e$ with excellent regio- and enantioselectivity.

A P450pyr double mutant I83M/I82T was also engineered to enable highly regioselective terminal hydroxylation of $n$-butanol 11 to afford 1,4-butanediol 12.

Several novel, accurate, sensitive, simple, and HTS assays based on colorimetric or MS detection for measuring the enantioselectivity and/or regioselectivity of hydroxylation were developed and proven to be practical in the directed evolution. These assays could be useful for the discovery of other chemical and/or bio-catalysts for regio- and/or enantioselective reactions.

The X-ray structure of P450pyr was determined and successfully used to identify the key amino acid residues for the evolutions. Molecular modelling on the hydroxylations of different substrates with P450pyr and P450pyr mutants gave some insight into the understanding of the roles of several key amino acid mutations for the enhanced enantioselectivity, reverted enantioselectivity, altered regioselectivity, and/or new substrate acceptance. This information and knowledge could be useful for further evolution of P450pyr to achieve high catalytic performance for other desired hydroxylations or oxidations.

\section{Acknowledgements}

Financial support by GlaxoSmithKline (GSK) and Singapore Economic Development Board (EDB) through Green and Sustainable Manufacturing Grant (project No. 279-000331-592) as well as Science and Engineering
Research Council of A*STAR of Singapore through Public Sector Research Funding (Project No. 0621010024) are gratefully acknowledged.

Received: January 26, 2015

Revised: February 6, 2015

[1] D. J. C. Constable, P. J. Dunn, J. D. Hayler, G. R. Humphrey, J. L. Leazer, Jr., R. J. Linderman, K. Lorenz, J. Manley, B. A. Pearlman, A. Wells, A. Zaks, T. Y. Zhang, Green Chem. 2007, 9 , 411; Z. Li, D. L. Chang, Curr. Org. Chem. 2004, 8, 1647; R. N. Patel, Coord. Chem. Rev. 2008, 252, 659; J. C. Lewis, P. S. Coelho, F. H. Arnold, Chem. Soc. Rev. 2011, 40, 2003

[2] J. C. Lewis, F. H. Arnold, Chimia 2009, 63, 309; G. D. Roiban, R. Agudo, M. T. Reetz, Angew. Chem. Int. Ed. 2014, 53, 8659; K. Kamata, K. Yonehara, Y. Nakagawa, K. Uehara, N. Mizuno, Nat. Chem. 2010, 2, 478 .

[3] N. M. Kamerbeek, D. B. Janssen, W. J. H. van Berkel, M. W. Fraaije, Adv. Synth. Catal. 2003, 345, 667; W. J. H. van Berkel, N. M. Kamerbeek, M. W. Fraaije, J. Biotechnol. 2006, 124, 670; Z. Li, J. B. van Beilen, W. A. Duetz, A. Schmid, A. de Raadt, H. Griengl, B. Witholt, Curr. Opin. Chem. Biol. 2002, 6, 136.

[4] I. G. Denisov, T. M. Makris, S. G. Sligar, I. Schlichting, Chem. Rev. 2005, 105, 2253; E. O'Reilly, M. Corbett, S. Hussain, P. P. Kelly, D. Richardson, S. L. Flitsch, N. J. Turner, Catal. Sci. Technol. 2013, 3, 1490.

[5] L. L. Wong, Curr. Opin. Chem. Biol. 1998, 2, 263; C. J. C. Whitehouse, S. G. Bell, L. L. Wong, Chem. Soc. Rev. 2012, 41, 1218; K. Y. Choi, E. Jung, D. H. Jung, B. P. Pandey, H. Yun, H. Y. Park, R. J. Kazlauskas, B. G. Kim, FEBS J. 2012, 279, 1650; E. O'Reilly, V. Kohler, S. L. Flitsch, N. J. Turner, Chem. Commun. 2011, 47, 2490; D. J. Koch, M. M. Chen, J. B. van Beilen, F. H. Arnold, Appl. Environ. Microbiol. 2009, 75, 337; M. T. Reetz, J. Am. Chem. Soc. 2003, 135,12480 .

[6] S. Kadkhodayan, E. D. Coulter, D. M. Maryniak, T. A. Bryson, J. H. Dawson, J. Biol. Chem. 1995, 270, 28042.

[7] M. W. Peters, P. Meinhold, A. Glieder, F. H. Arnold, J. Am. Chem. Soc. 2003, 125, 13442; R. Lauchli, K. S. Rabe, K. Z. Kalbarczyk, A. Tata, T. Heel, R. Z. Kitto, F. H. Arnold, Angew. Chem., Int. Ed. 2013, 52, 5571.

[8] T. L. Poulos, B. C. Finzel, A. J. Howard, J. Mol. Biol. 1987, 195, 687; K. G. Ravichandran, S. S. Boddupalli, C. A. Hasemann, J. A. Peterson, J. Deisenhofer, Science 1993, 261, 731; I. Schlichting, J. Berendzen, K. Chu, A. M. Stock, S. A. Maves, D. E. Benson, B. M. Sweet, D. Ringe, G. A. Petsko, S. G. Sligar, Science 2000, 287, 1615; A. Li, S. Wu, J. P. Adams, R. Snajdrova, Z. Li, Chem. Commun. 2014, 50, 8771.

[9] M. T. Reetz, D. Kahakeaw, R. Lohmer, ChemBioChem 2008, 9, 1797; K. L. Tee, U. Schwaneberg, Comb. Chem. High. Throughput. Screen. 2007, 10, 197; T. Davids, M. Schmidt, D. Bottcher, U. T. Bornscheuer, Curr. Opin. Chem. Biol. 2013, 17, 215; U. T. Bornscheuer, G. W. Huisman, R. J. Kazlauskas, S. Lutz, J. C. Moore, K. Robins, Nature 2012, 485, 185; H. Zhao, Biotechnol. Bioeng. 2007, 98, 313; N. J. Turner, Nat. Chem. Biol. 2009, 5, 568.

[10] A. Dennig, N. Lulsdorf, H. Liu, U. Schwaneberg, Angew. Chem. Int. Ed. 2013, 52, 8459.

[11] C. F. Harford-Cross, A. B. Carmichael, F. K. Allan, P. A. England, D. A. Rouch, L. L. Wong, Protein Eng. 2000, 13, 121; S. Kumar, C.S. Chen, D. J. Waxman, J. R. Halpert, J. Biol. Chem. 2005, 280, 19569; J. D. Bloom, F. H. Arnold, Proc. Natl. Acad. Sci. 2009, 106, 9995. 
[12] M. T. Reetz, Proc. Natl. Acad. Sci. 2004, 101, 5716; M. Landwehr, L. Hochrein, C. R. Otey, A Kasrayan, J. E. Backvall, F. H. Arnold, J. Am. Chem. Soc. 2006, 128, 6058; R. E. Cobb, T. Si, H. Zhao, Curr. Opin. Chem. Biol. 2012, 16, 285.

[13] S. Kille, F. E. Zilly, J. P. Acevedo, M. T. Reetz, Nat. Chem. 2011, 3, 738

[14] K. Zhang, B. M. Shafer, M. D. Demars. II, H. A. Stern, R. Fasan, J. Am. Chem. Soc. 2012, 134, 18695.

[15] D. Chang, B. Witholt, Z. Li, Org. Lett. 2000, 2, 3949; Z. Li, H. J. Feiten, D. Chang, W. A. Duetz, J. B. van Beilen, B. Witholt, J. Org. Chem 2001, 66, 8424; D. L. Chang, H. J. Feiten, K. H. Engesser, J. B. van Beilen, B. Witholt, Z. Li, Org. Lett. 2002, 4, 1859; D. L. Chang, H. J. Feiten, B. Witholt, Z. Li, Tetrahedron Asymmetr. 2002, 13, 2141; W. Zhang, W. L. Tang, Z. S. Wang, Z. Li, Adv. Synth. Catal. 2010, 352, 3380

[16] Z. Li, H. J. Feiten, J. B. van Beilen, W. Duetz, B. Witholt, Tetrahedron Asymmetr. 1999, 10, 1323.

[17] W. L. Tang, Z. Li, H. M. Zhao, Chem. Commun. 2010, 46, 5461

[18] J. K. Yano, L. S. Koo, D. J. Schuller, H. Li, P. R. Ortiz de Montellano, T. L. Poulos, J. Biol. Chem. 2000, 275, 31086; Y. Oku, A. Ohtaki, S Kamitori, N. Nakamura, M. Yohda, H. Ohno, Y. Kawarabayasi, J. Inorg. Biochem. 2004, 98, 1194.

[19] M. T. Reetz, J. D. Carballeira, Nat. Protoc 2007, 2, 891.

[20] M. T. Reetz, J. Org. Chem. 2009, 74, 5767.

[21] Z. Li, L. Butikofer, B. Witholt, Angew. Chem. Int. Ed. 2004, 43, 1698.
[22] N. Kizaki, Y. Yasohara, J. Hasegawa, US 07033808, 2006

[23] N. Kizaki, Y. Yasohara, N. Nagashima, J. Hasegawa, J. Mol. Catal. B Enzym. 2008, 51 73.

[24] R. Woodyer, W. A. van der Donk, H. M. Zhao, Biochemistry US. 2003, 42, 11604.

[25] S. Q. Pham, G. Pompidor, J. Liu, X. D. Li, Z. Li, Chem. Commun. 2012, 48, 4618.

[26] Y. Z. Chen, W. L. Tang, J. Mou, Z. Li, Angew. Chem. Int. Ed. 2010, 49, 5278.

[27] S. Q. Pham, P. Gao, Z. Li, Biotechnol. Bioeng. 2013, 110, 363.

[28] A. Li, J. Liu, S. Q. Pham, Z. Li, Chem. Commun. 2013, 49, 11572 .

[29] P. Gao, A. Li, H. H. Lee, D. I. C. Wang, Z. Li, ACS Catal. 2014, 4, 3763.

[30] J. A. Labinger, J. E. Bercaw, Nature 2002, 417, 507.

[31] D. Böttcher, U. T. Bornscheuer, Curr. Opin. Microbiol. 2010, 13, 274; D. Scheps, S. Honda Malca, H. Hoffmann, B. M. Nestl, B. Hauer, Org. Biomol. Chem. 2011, 9, 6727.

[32] E. T. Farinas, U. Schwaneberg, A. Glieder, F. H. Arnold, Adv. Synth. Catal. 2001, 343, 601

[33] K. H. Halsey, L. A. Sayavedra-Soto, P. J. Bottomley, D. J. Arp, J. Bacteriol. 2006, 188, 4962.

[34] P. Meinhold, M. W. Peters, M. M. Chen, K Takahashi, F. H. Arnold, ChemBioChem 2005, 6, 1765; A. Glieder, E. T. Farinas, F. H. Arnold, Nat. Biotechnol. 2002, 20, 1135.

[35] S. G. Bell, J. A. Stevenson, H. D. Boyd, S. Campbell, A. D. Riddle, E. L. Orton, L. L. Wong, Chem. Commun. 2002, 490.
[36] Liese, T. Zelinski, M.-R. Kula, H. Kierkels, M. Karutz, U. Kragl, C. Wandrey, J. Mol. Catal. B 1998, 4, 91.

[37] Y. Hu, E. C. K. Lin, L. M. Pham, J. Cajica, C. M. Amantea, E. Okerberg, H. E. Brown, A. Fraser, L. Du, Y. Kohno, J. Ishiyama, J. W. Kozarich, K. R. Shreder, Bioorg. Med. Chem. Lett. 2013, 23, 1553.

[38] H. Yamamoto, K. Kawada, A. Matsuyama, Y. Kobayashi, Biosci. Biotech. Bioch. 1999, 63 , 1051.

[39] H. Yamamoto, M. Kudoh, Appl. Microbiol. Biotechnol. 2013, 97, 8087.

[40] O. Lentz, A. Feenstra, T. Habicher, B. Hauer, R. D. Schmid, V. B. Urlacher, ChemBioChem 2006, 7, 345.

[41] J. M. Wagner, C. J. McElhinny, Jr., A. H. Lewin, F. I. Carroll, Tetrahedron Asymmetr. 2003, 14, 2119.

[42] S. K. Talluri, A. Sudalai, Tetrahedron 2007, 63, 9758.

[43] F. X. Tavares, D. N. Deaton, A. B. Miller, L. R. Miller, L. L. Wright, Bioorg. Med. Chem. Lett. 2005, 15, 3891

[44] Y. Yang, J. Liu, Z. Li, Angew. Chem. Int. Ed. 2014, 53, 3120.

[45] M. Barikani, H. Honarkar, M. Barikani, J. Appl. Polym. Sci. 2009, 112, 3157; L. J. Schep, K. Knudsen, R. J. Slaughter, J. A. Vale, B. Megarbane, Clin. Toxicol. 2012, 50, 458; M. Seitz, O. Reiser, Curr. Opin. Chem. Biol. 2005, 9, 285.

[46] P. Meinhold, M. W. Peters, A. Hartwick, A. R. Hernandez, F. H. Arnold, Adv. Synth. Catal. 2006, 348, 763.

[47] Y. Yang, Y. T. Chi, H. H. Toh, Z. Li, Chem. Commun. 2015, 51, 914 